\title{
A Intelligent Electronic Device(IED) Used In Active Distribution Network Hai HUANG ${ }^{a^{*}}$, Qiu-Wei CAO ${ }^{b}$, Wei-Hong LI ${ }^{c}$, Xin-Xin GU NJ GRID RELAYS Co. Ltd.,No.20 Lijing Road, Pukou District Nanjing 210032, China

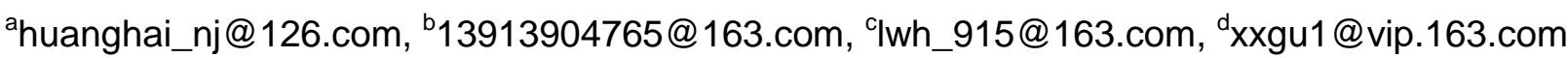 ${ }^{*}$ Corresponding author
}

Keywords: Active Distribution Network, Differential Protection, IED; EPON, synchronous sampling.

\begin{abstract}
The development of active power distribution network control and automation system presents the trend of distributed control. The basic work of the system mainly depends on the information collection, transmission and control function of distributed Intelligent Electronic Device(IED). Protection, control and monitoring of the depth of the integration of IED, collectively referred to as the active power distribution network intelligent terminal. Distribution network in our country communications are established widely adopted Ethernet Passive Optical Network(EPON), If the intelligent terminal of active power distribution network is to meet the precision of synchronous sampling of current differential protection, often need external measures for time synchronization, For example, to use GPS or the IEEE 1558 protocol software. Fault state differential protection can be used to simplify the time synchronization, which is more suitable for Ethernet communication in EPON. In this paper, we propose of the synchronization method using a voltage zero crossing, to realize fault state differential protection.
\end{abstract}

\section{Summary}

Traditional distribution network has single direction of load flow, namely, flowing from generation side to power utilization side. The access of distributed generation, electric vehicle and charging station to the distribution network changes the nature of load flow, making the network transfer into multi-terminal and active network from passive network. As the penetration rate of new energy rises, the existing distribution network becomes increasingly complex.

The large-scale of distributed energy resources with high penetration rate will have impact on the global electric power system, especially the planning, operation, device selection, control and automation system [1]. To face the challenge in the future, China has made great efforts to concentrate on technology research and application demonstration in order to transfer China traditional distribution network into active distribution network.

\section{Key Devices to Active Distribution Network}

The key devices of active distribution network falls broadly into two categories, hardware and software. Wherein, hardware includes distribution network flexible alternative current transmission systems (D-FACTS), Intelligent Electronic Device(IED), communication device, energy storage device, coordinated control equipment access to distributed power supply; Software includes Energy Management System, Distribution Management System, Distributed Energy Resource (DER) system and demand side management system.

Since traditional distribution network was not originally designed for large-scale access of DER, the large-scale access of DER may have a lot of influences in other aspects. For instance, level of short circuit, reactive power and voltage distribution are affected. The plug-and-play way of accessing new energy increases the uncertainty of distribution network, making it more difficult to control. To solve the problem, distribution network had better develop into active control, selfadapting active distribution network and the technology roadmap for power supply units have to take measures to response to the changes. 


\section{Design Thinking for Key Devices of Active Distribution Network}

Completely different from design approaches of regular distribution network, intelligent equipment of active distribution network should be designed in the way of active control technique.

The devices for regular distribution network are selected according to single direction of load flow, short-circuit current level; The reactive compensation capacity is configured according to nature of loads, so the parameters are fixed, unable to regulate and control.

The active distribution network is designed according to power electronic, information collection, communication technologies and self-healing concept by ways of application wide-area information, data processing, type identification to realize the prediction, judgment, early intervention, load transfer, to decrease the probabilities of failure as much as possible, ensuring highly reliable operation. On one hand, apply D-FACTS and flexible AC transmission technique to regulate voltage and reactive and take the initiative to control voltage stability; On the other hand, apply distributed control strategy, the setting decentralized local control and global centralized control to establish methods to deal with emergency conditions, failure conditions, restoration state of power system and a series of logic control method and setting up active control system.

\section{The Development of IED Fault Isolation}

Protective, controlling and monitoring equipment are deeply integrated and called Intelligent Electronic Devices (IED) of active distribution network. They have the following features at least:

Collect, control and execute distributed control system, support network technique and collect wide-area information.

Adopt protection, logic control and setting values self-adaptive technology and self-adapt to the operation conditions of distribution network, the changes in network topology and seek for the optimum sensitivity of protection, speedability and reliability.

\section{IED Based on Active Control Design Concept}

(1)Self-adaptive relay protection

For IED developed with graphic editing logic programmatic hardware and software platform, its relay protection functions can be reconfigured with the flexible changes in the distribution network topological structure. And, the active control of the multiple self-adaption, multi-point, multisurface network protection and control will be the mainstream research and development and design concept.

(2)Self-adaptive control

The remote trip, remote backup power automatic switching, remote load regulating, power supply, network and loads coordinating control equipment and control system in areas with high density of new energy access will meet specific requirements to adapt to access and changed new energy at any time and to the changes in network topology.

The following distribution network area mounted with distributed active distribution network terminal IED is to describe the benefits deeply integrated IED has brought to controlling and protection system of active distribution network. It enables multiple isolation and reconstitution. 


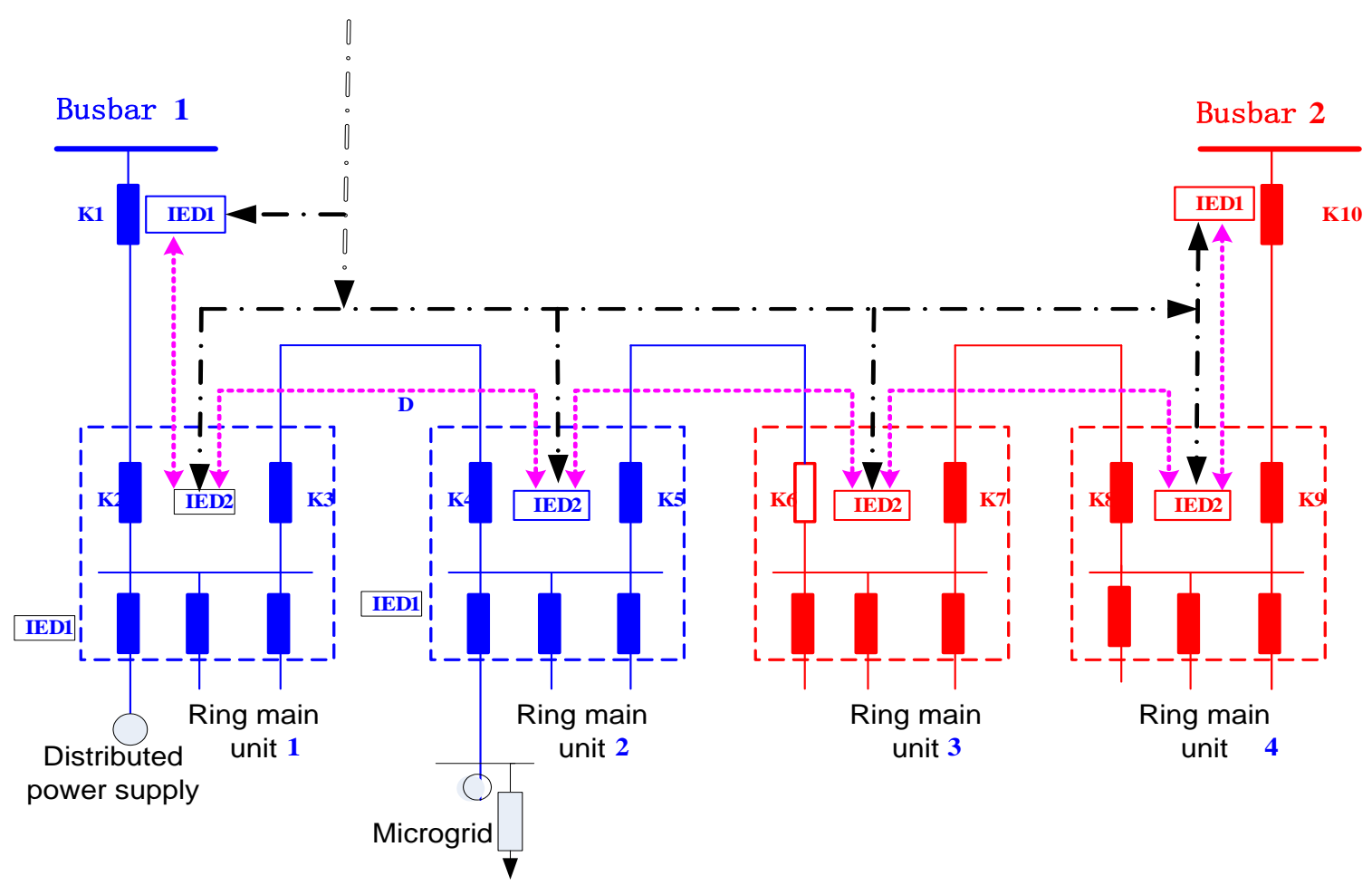

Fig.1 IED distributed acquisition and control system.

As Fig. 1 shows:

- Blue busbar and red busbar supply power to the supply district, respectively.

- IED1 is arranged at substation terminal, for collecting conditions and analog information of switches K1 and K10.

- IED2 collects condition and analog information about ring main unit or dual incoming line switch.

Active distribution network supports flexible network topology. When network topology changes, the master station will issue new topology structure and establish new correlation relation for IED.

\section{Distributed IED Composes Various Failure Isolation Modes}

The IED highly integrated with protection, control and communication systems can compose various control modes after the completion of collection.

(1)Local feeder automation adjacent point to point IED communication can realize rapid fault localization, isolation, isolating faulty section, rapid recovery of power supply in non-faulty zone; Cut off failure are before outlet breaker acts and make zero power outage possible. After the recovery of power supply, IED will report the treatment conditions and topology structure to master station.

(2) Master station centralized feeder automation

Distributed IED collects and reports information into distribution master station and the advanced application software in master station will handle failures by fault treatment logic in order to realize isolation fault, rapid recovery and send down results to IED.

(3)Current or current direction differential protection

Current differential protection is one of local feeder automation and it is well adapted to coordinate with flexible net rack. For instance, the supply network of petals type connection mode that has rapidly expended over the years is very fit for differential protection. At present, however, EPON applied in distribution automation, if not technically improved, will lead to low availability of current differential protection [4]. If current differential protection is used to transfer current sampling value, timing measure 1588 should be added at least. [2][3]. EPON communication 
network covers almost most parts of China, so the existing network resources should be taken into consideration in the process of configuring IED. Due to the huge amount of work, technological and economic barriers, it is almost impossible to mount distribution network with point-to-point communication network usually used in relay protection.

EPON system consists of centered Optical Line Terminal (OLT), Optical Network Unit(ONU) at consumption side and passive optical splitter . EPON transfers upstream and downstream waves via a fiber core. At the direction of downstream (from OLT to ONU), the signal from OLT will reach each ONU through 1: n passive optical splitter (or a cascade of several optical splitter); At the direction of upstream (from ONU to OLT), signals from ONU reach OLT only, instead of other ONUs. To avoid data contention and improve the utilization efficiency of network, the time division multiple address access method will be applied at upstream direction and mediation of data deliver to ONU is made through OLT.

EPON uses wavelength division multiplexing method to realize two-way transmission of data via one optical fiber by taking advantage of different wavelength. At downstream direction, using $1490 \mathrm{~nm}$ wavelength and broadcast technology, the signals from OLT are broadcast to each ONU via passive optical splitter. At upstream direction, using $1310 \mathrm{~nm}$ wavelength and the time division multiple address access technology, ONU will upload the signals to be sent to OLT within the specified time slot. In case the data exchange between ONUs is required, it should be forwarded via OLT, instead of direct communication.

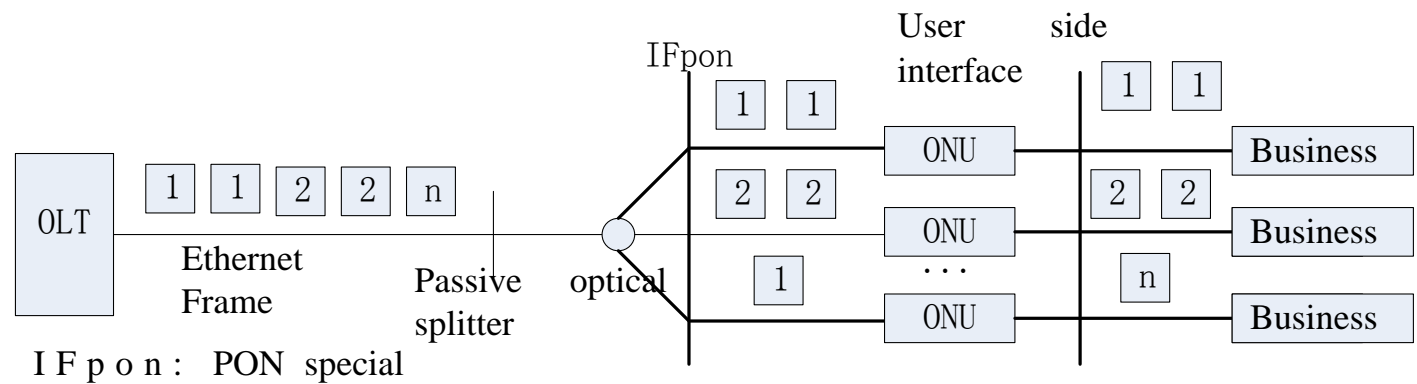
interface

Fig.2 PON transmit upstream channel.

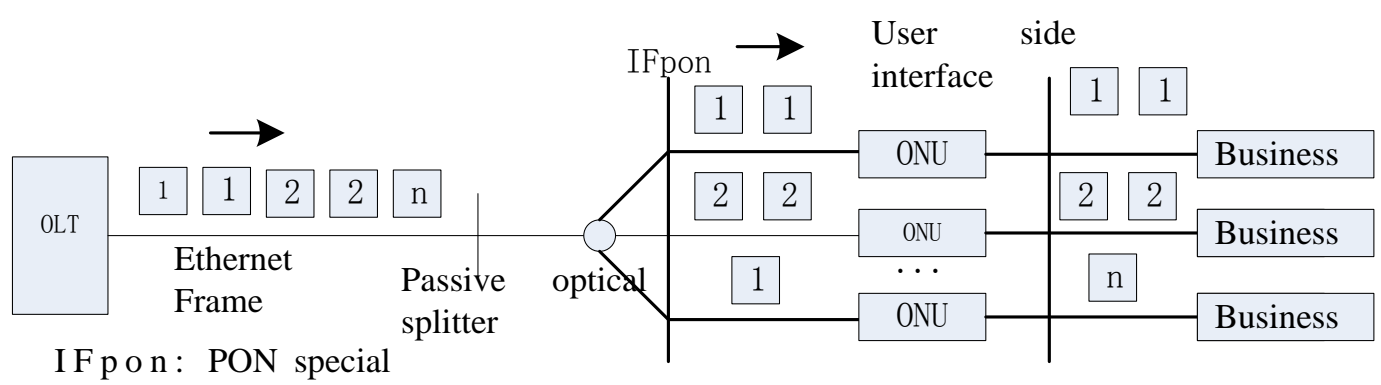

interface

Fig.3 ownstream channel for EPON transmission.

Fig 2 and Fig 3 represent the mode how EPON system data works at upstream and downstream. EPON uses wavelength division multiplexing method to realize two-way transmission of data via one optical fiber by taking advantage of different wavelength. At downstream direction, using 1490nm wavelength and broadcast technology, the signals from OLT are broadcast to each ONU via passive optical splitter. At upstream direction, using $1310 \mathrm{~nm}$ wavelength and time division multiple address access technology, ONU will upload the signals to be sent to OLT within the specified time slot. In case the data exchange between ONUs is required, it should be forwarded via OLT, instead of direct communication.

Fig 2 and Fig 3 show that EPON network time delay is divided into up, downstream direction. downstream direction is relatively simple, OLT via a broadcast data that is sent to the ONU, the 
network delay is mainly OLT, ONU hardware processing delay and fiber channel transmission delay. The former is relatively small and fixed; For the latter, according to 802.3ah [5] of IEEE, max physical transmission distance for EPON is $20 \mathrm{~km}$ and the time delay rate is $5 \mu \mathrm{s} / \mathrm{km}$, so the delayed time is $100 \mu \mathrm{s}$, which is fixed. The sum of the two values is the fixed time delay at downstream direction. It is relatively complex of upstream to calculate time delay. ONU needs to apply to OLT for time slot first and then produce time delay for waiting before transmission; ONU needs to queue up for data transmission and produce time delay for data queuing by priority of business within the specified time slot; Apart from it, there is fixed time delay corresponding to that in down direction. Time delay for waiting before transmission and data queuing are variable time delay characterized by Ethernet. As data traffic grows the time delay for transmission will increase and delay jitter greatly changes, which have bad influence on real-time and reliability of transmission and may lead to mal-operation of protective relay.

Literature [4] provides the important measures for smart distribution network optical differential business EPON carrying solution, including expanding bandwidth control: set the max static bandwidth and improve dynamic bandwidth allocation plan; increasing synchronization measures: realize synchronization of OLT and ONU in accordance with IEEE 802.1as and realize synchronization of sampling in of optical differential protection accordance with IEEE 1588v2. After the EPON network technically improved, current differential protection overcomes time delay jitter and increases time delay to a little bit longer than action time of differential protection that applies point-point communication mode, making current differential protection come true.

Differential protection for failure state is introduced so that only status information is transmitted between adjoining IEDs. Unlike transmitting sampling value, each sampling point is not necessarily strictly synchronous. What's more, the data is relatively short, so it is more suitable for EPON mode of transmission.

As shown in Figure 1,in the distributed IED system, IED at each node has a correlation with the adjoining IED only and the displaying or not displaying current value, input or output in direction of current can be expressed by $0,1,-1$ in numbers. Only transmission of status message is necessary for adjoining IEDs and requirements for synchronization are much less. Within the interval time of mastering station timing, IED automorph time is not more than a cyclic wave. Assisted with synchronization at voltage zero crossing point of each cyclic wave, requirement for differential protection failures is satisfied.

(4)Fault analysis

Since each IED needs to exchange information with nearby IED only, the relation between neighboring switches are called adjoining vector table.

Tab.1 Adjoining Vector Table.

\begin{tabular}{cc}
\hline Switch number & Adjoining switch number \\
\hline 1 & $(2)$ \\
2 & $(1,3)$ \\
3 & $(2,4)$ \\
4 & $(3,5)$ \\
5 & $(4,6)$ \\
6 & $(5,7)$ \\
7 & $(6,8)$ \\
8 & $(7,9)$ \\
9 & $(8,10)$ \\
10 & $(9)$ \\
\hline
\end{tabular}

Each IED is offered with a failure volume "n" to reflect whether online current value is out-oflimit. When faulty current is larger than the set value, it is out-of-limit. The faulty state variable is expressed as 1 , otherwise 0 . When the fault occurs, the IED faulty state variables turn into 1 or there 
is voltage loss to circuit. At the same time, the IED would automatically transmit state variables, switch state and recorded signals from one end into the adjoining IED. See table 2.

Tab.2 Information table to pass to adjacent IED.

\begin{tabular}{cccc}
\hline Switch number & $\mathrm{k}$ & $\mathrm{k}-1$ & $\mathrm{k}+1$ \\
Failure volume & $\mathrm{N}_{\mathrm{k}}$ & $\mathrm{N}_{(\mathrm{k}-1)}$ & $\mathrm{N}_{(\mathrm{k}+1)}$ \\
\hline
\end{tabular}

The state vector of each IED is provided as criteria for trip protection. State vector table for each IED is recorded in faulty state differential protection scheme. The first line in table 2 shows IED number and the adjoining IED number, which is determined by system structure. Data is issued by master station. The second line in Table 2 is obtained through communication between IED and adjoining IED and provided as criteria for determining faults. The self-trouble state variable is considered the condition for the IED starting protection.

(5)Criteria

Faulty current is usually flowing through two sides of substations to the faulty point In case of single fault, the faulty section is certain to be between the last switch experiencing forward direction faulty current and the last switch experiencing negative faulty current from substation's side to the faulty side. The switch at the faulty end will feel the over-current, and the adjoining switch will experience faulty current in forward direction while the other switch will experience faulty current in negative direction; For the switch opposite to faulty point, one adjoining switch (upstream switch) will experience faulty current in forward direction while the other switch (downstream switch) will experience faulty current in negative direction; For switches flowing through faulty current, the adjoining switches experience faulty current in both directions. IED criteria for outlet switches 1,10 should be treated in different ways.

Assume vector J:

$\mathrm{J}=(\mathrm{S}, \mathrm{H}, \mathrm{L})$

Wherein,"S" stands for over-current state and various detection conditions are as follows:

When over-current in forward direction is detected, it is set as "1"; when over-current in negative direction is detected, it is set as "-1"; when no over-current is detected, it was set as "0". H, indicating over-current state of upstream switch, is set as "1" when receive over-current information of upstream switch in forward direction; as "-1" when receive over-current information of upstream switch in negative direction; as "0" when no over-current information about upstream switch is received. $\mathrm{L}$, indicating over-current state of downstream switch, is set as "1" when receive overcurrent information of downstream switch in forward direction; as "-1" when receive over-current information of downstream switch in negative direction; as "0" when no over-current information about downstream switch is received.

Criteria for failures are:

Judging conditions of ordinary switch at end of failure are:

$\mathrm{J}=(\mathrm{x}, 1,-1)$

The conditions for judging faulty outlet switch in upstream side substation are:

$\mathrm{J}=(1, \mathrm{y},-1)$

The conditions for judging faulty outlet switch in downstream side substation are:

$\mathrm{J}=(-1,1, \mathrm{y})$

Whereby, $\mathrm{x}$ is placed with 1 or -1 , while $\mathrm{y}$ is set Unknown or criteria unconcerned. 
Tab.3 Fault state differential protection action criterion vector table.

\begin{tabular}{cccc}
\hline S & H & L & Action \\
1 & 1 & 1 & Immobile \\
-1 & -1 & -1 & Immobile \\
1 & -1 & -1 & Trip \\
1 & 1 & -1 & Trip \\
0 & 0 & 0 & Immobile \\
\hline
\end{tabular}

\section{Adapt to Flexible Net Rack}

The net rack of petals type connection mode is substation-centered wiring form in shape of petal, that is to say, in the substation with the same dual power transformers parallel running, a ring net is formed by every two feeders and it is running in a closed loop appearance of petal, as shown in Figure 4; Interconnection switches are installed in the petal between different power substations, and they are in normal open-loop operation condition.

For this flexible net rack fault condition, the differential protection applies. IED with contact switches designed with backup power supply automatic switch-in equipment between petals can constitute rapid reconfiguration control.

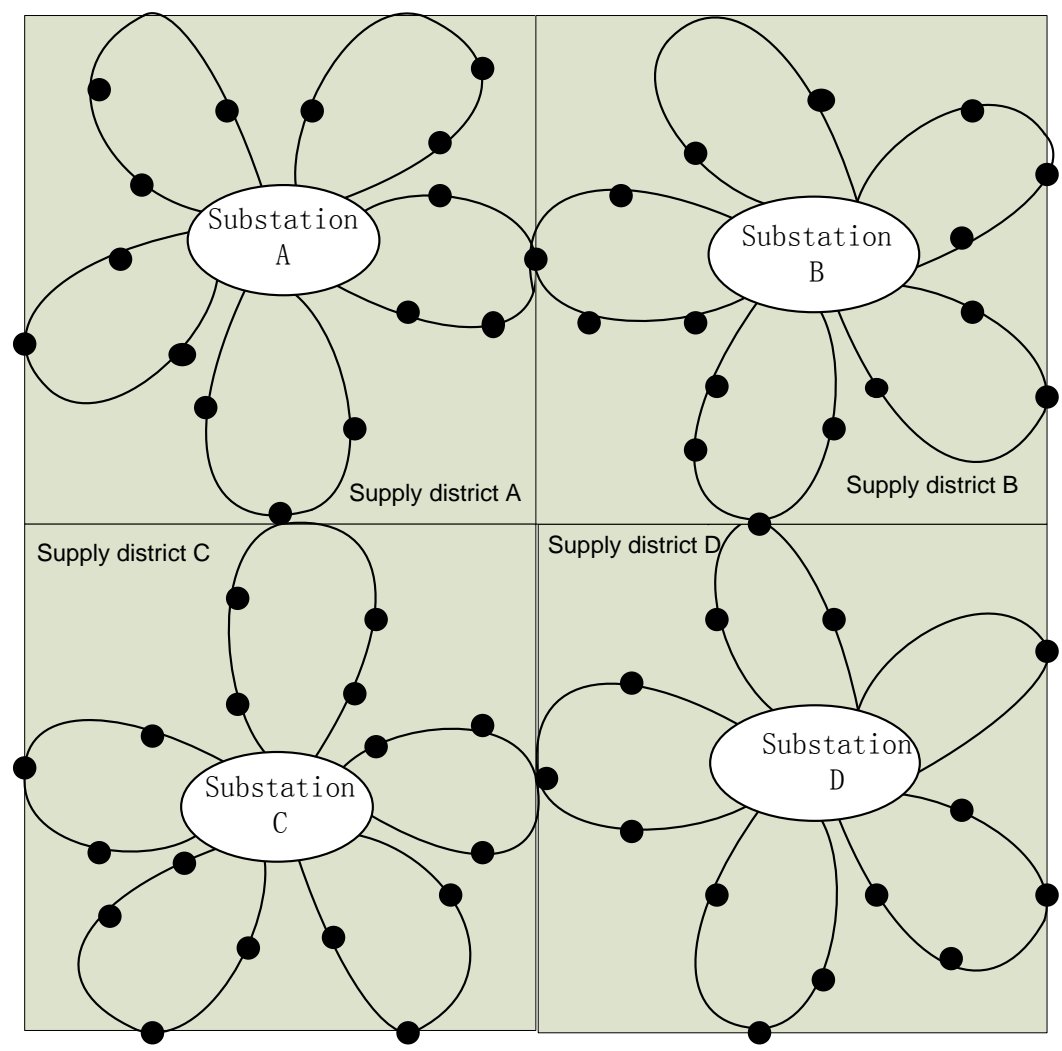

Fig.4 Net rack of petals type connection mode.

\section{Conclusion}

Protective, control and monitoring equipment for active distribution network will be deeply integrated and are collectively called intelligent electronic device (IED) of active distribution network. IED undertakes the tasks of collecting, controlling, and protecting distributed control system, supports network technology and collects wide area information.

IED is configurable with commonly used protective features. we recommend using the existing communications network does not make excessive demands, try to reduce secondary transformation 
and construction. It should be a protection and control method with economical and practical, highly reliable, technologically advanced. Fault differential status protection is a good choice.

\section{References}

[1] Fan Mingtian, Zhang Zuping, et al, Study on feasibility of active power distribution system [J]. Proceedings of the CSEE Vol.33, No.22, Aug. 5. 2013.

[2] Li Ruisheng, Research into differential protection adaptive to active distribution network, Power System Protection and Control, Vol.43, No.12, Jun.16, 2015.

[3] Fu Guangfu, Zhang Chunhe,Yan Wei eta al, EPON communication-based intelligent distribution feeder differential protection [J]. Power system automation, 2014, 38(2): 91-96.

[4] Qiu Yutao, Zhu Ma, et al, Study on EPON carrying solution of intelligent distribution network optical differential protection business [J]. China Electic Power Technology, Issue.9, 2014.

[5] IEEE 802.3ah, Ethernet in the First Mile Task Force[S].2004. 